\title{
Analysis of the frequency-dependent response to wave forcing in the extratropics
}

\author{
A. J. Haklander ${ }^{1,2}$, P. C. Siegmund ${ }^{2}$, and H. M. Kelder ${ }^{1,2}$ \\ ${ }^{1}$ Eindhoven University of Technology (TUE), Department of Applied Physics, P.O. Box 513, 5600 MB Eindhoven, \\ The Netherlands \\ ${ }^{2}$ Royal Netherlands Meteorological Institute (KNMI), Climate Research and Seismology Department, Atmospheric \\ Composition Division, P.O. Box 201, 3730 AE De Bilt, The Netherlands
}

Received: 25 November 2005 - Published in Atmos. Chem. Phys. Discuss.: 20 February 2006

Revised: 3 May 2006 - Accepted: 4 October 2006 - Published: 5 October 2006

\begin{abstract}
A quasigeostrophic model for the frequencydependent response of the zonal-mean flow to planetarywave forcing at Northern Hemisphere $(\mathrm{NH})$ midlatitudes is applied to 4-D-Var ECMWF analysis data for six extended winter seasons. The theoretical response is a non-linear function of the frequency of the forcing, the thermal damping time $\alpha^{-1}$, and a scaling parameter $\mu$ which includes the aspect ratio of the meridional to the vertical length scale of the response. Regression of the calculated response from the analyses onto the theoretical response yields heightdependent estimates for both $\alpha^{-1}$ and $\mu$. The thermal damping time estimated from this dynamical model is about 2 days in the troposphere, 7-10 days in the stratosphere, and 2-4 days in the lower mesosphere. For the stratosphere and lower mesosphere, the estimates lie within the range of existing radiative damping time estimates, but for the troposphere they are significantly smaller.
\end{abstract}

\section{Introduction}

In $\mathrm{NH}$ midlatitude winter, planetary waves strongly interact with the zonal-mean flow. This interaction occurs at a broad range of timescales. For instance, the intense growth of a sudden stratospheric warming (SSW) event can occur in just a few days, its mature stage typically lasts a few weeks, and the total SSW cycle from preconditioning to recovery of the polar vortex can last a few months (e.g., Limpasuvan et al., 2004). Randel et al. (2002) found that the power spectrum of equatorial upwelling shows a broad maximum at periods between 10-40 days, and they argued that this is associated with episodes of planetary-wave activity at midlatitudes. The

Correspondence to: A. J. Haklander

(haklande@knmi.nl) midlatitude zonal forcing by planetary waves also exhibits an annual cycle (e.g., Rosenlof, 1995). During winter, westerly stratospheric winds allow the vertical propagation of planetary waves emanating from the troposphere, whereas the summertime easterlies block the planetary waves. The angular momentum that is deposited by the waves is distributed between the relative and planetary reservoirs of angular momentum (e.g., Peixoto and Oort, 1992). This is expressed by the quasigeostrophic transformed Eulerian mean (TEM) zonal momentum equation,

$\frac{\partial \bar{u}}{\partial t}-f \overline{v^{*}}=\bar{M}$,

where the overbars denote zonal averages, $\bar{u}$ is the zonalmean zonal wind, $f$ is the Coriolis parameter, $\overline{v^{*}}$ is the meridional component of the residual circulation, and $\bar{M}$ is $a \cos \phi$ times the wave-induced angular momentum deposition per unit mass (e.g., Holton et al., 1995). The tendency of the relative angular momentum is described by $\partial \bar{u} / \partial t$, that of the planetary angular momentum by $-f \overline{v^{*}}$. Garcia (1987) derived an expression for the frequency dependence of the $\partial \bar{u} / \partial t$ response to a given zonal wave forcing $\bar{M}$. According to this expression, the wave-induced angular momentum deposition at high frequencies is distributed about equally between the relative and angular momentum reservoirs. It was found that, away from the high-frequency limit, the rates of frictional dissipation and thermal relaxation toward radiative equilibrium determine the frequency dependence of the response. The timescale of thermal damping is important, since for non-zero $\partial \bar{u} / \partial t$ it determines how efficiently the meridional circulation is able to restore thermal-wind equilibrium through the adiabatic adjustment of the meridional temperature gradient. In the low-frequency limit, which defines the Brewer-Dobson circulation, the thermal response to the wave forcing is fully damped by thermal relaxation,

Published by Copernicus GmbH on behalf of the European Geosciences Union. 
implying that the angular momentum of the waves is deposited into the planetary reservoir only. Although Garcia's model provides valuable insights into the frequency dependence of the balance in Eq. (1), it has not been tested with real atmospheric data. In this study, we apply this model to wind and temperature analyses from the ECMWF model. Since the stratospheric diabatic heating during summer is no longer slaved to the wave forcing, we base our analysis on $\mathrm{NH}$ winter (November to April) data only. For each of the three terms in Eq. (1), power spectra estimates are computed, using six winters of 12-hourly analysis data. Estimates for the vertical profiles of the thermal damping time and the high-frequency limit of the response are obtained, using a least-squares method to fit the theoretical frequency dependence of the response of $\partial \bar{u} / \partial t$ to $\bar{M}$ onto the analyzed data. It should be emphasized that we are estimating the "effective" thermal damping time, which in the troposphere is significantly shorter than the radiative damping time (e.g., Wu et al., 2000). The thermal damping times derived in the present study are assumed to represent all thermal dissipative processes, such as turbulent heat transfer and convection.

\section{Frequency-dependent TEM model}

In the TEM model used by Garcia (1987), infrared cooling is parameterized by Newtonian cooling with thermal damping rate $\alpha$, and gravity-wave drag is parameterized by Rayleigh friction with coefficient $K_{R}$. The forcing is assumed to be entirely due to the Eliassen-Palm (E-P) flux divergence of planetary waves, and thereby the forcing due to shortwave radiative heating is neglected. To study the frequency dependence of the zonal-mean flow response, a harmonic wave forcing at angular frequency $\omega$ is assumed, i.e., $\bar{M}=\operatorname{Re}\left(\bar{M}_{\omega} e^{i \omega t}\right)$. The zonal-mean zonal wind field can then be written as $\bar{u}=\operatorname{Re}\left(\bar{u}_{\omega} e^{i \omega t}\right)$. Garcia (1987) showed that the complex amplitude $i \omega \bar{u}_{\omega}$ of the zonal-mean zonal wind tendency Fourier component with angular frequency $\omega$ can be scaled as

$i \omega \bar{u}_{\omega} \sim \mu \frac{(1+\mu)+(\alpha / \omega) i}{(1+\mu)^{2}+(\alpha / \omega)^{2}} \bar{M}_{\omega}$,

where $\mu$ is a real, non-dimensional scaling parameter defined as $\mu \equiv L_{z}^{2} N^{2} /\left(L_{y} f\right)^{2}$, which is typically $\mathrm{O}(1)$ in the extra-tropics. $N^{2}$ denotes the square of the buoyancy frequency, and $L_{y}$ and $L_{z}$ denote the meridional and vertical scales of the response. Equation (2) is a rewritten form of the scaling argument found by Garcia (1987, Eq. 14), but with $K_{R}$ set to zero. Rayleigh friction introduces a nonphysical momentum sink (e.g. Shepherd and Shaw, 2004), and it is difficult to argue that a linear drag such as Rayleigh friction is at all relevant in the stratosphere (Haynes, 2005). For these reasons, we have not adopted this parameterization in the present study. To compare the magnitudes of the Fourier components, we multiply both sides of Eq. (2) with their complex conjugate. Subsequently taking the square root of both sides yields the response function $r$,

$$
\begin{aligned}
& \frac{\left|\partial \bar{u}_{\omega} / \partial t\right|}{\left|\bar{M}_{\omega}\right|} \sim r(\alpha, \mu, \omega) \\
& \quad \text { with } r(\alpha, \mu, \omega) \equiv \frac{\mu}{\sqrt{(1+\mu)^{2}+(\alpha / \omega)^{2}}} .
\end{aligned}
$$

In the high-frequency limit Eq. (3) converges to $\left(1+\mu^{-1}\right)^{-1}$, and in the stationary limit it converges to zero. Garcia (1987) set $\mu=1$, but we allow $\mu$ to be a function of height. As expressed by Eq. (1), $\partial \bar{u} / \partial t$ not only depends on the local $\bar{M}$ but on the entire forcing field, due to the Coriolis torque on the wave-driven meridional circulation. We realize that treating the scaling argument in Eq. (3) as an approximate equality between locally evaluated zonal-mean fields is a major simplification. However, the $\bar{M}$ evaluated at a particular location is expected to be a good guide to $\bar{M}$ over a significant region around that location. It is the latter $\bar{M}$ that mainly determines the local acceleration. A more precise extension of this work would be to take account of the non-local dependence of the acceleration on the forcing.

\section{Data and method}

For this study, we have used 12-hourly operational ECMWF four-dimensional variational (4-D-Var) analysis data for six NH winters (November to April) from 1999/2000 to $2004 / 2005$. The wind and temperature fields were retrieved for a latitude-longitude grid of $1^{\circ} \times 1^{\circ}$ at 35 pressure levels: $1000,925,850,700,500,400,300,250,200,150,100,70$, 66.6, 54.6, 44.3, 35.8, 28.9, 23.3, 18.8, 15.2, 12.3, 9.9, 8.0, $6.4,5.2,4.2,3.4,2.7,2.1,1.6,1.2,0.8,0.5,0.3$, and $0.1 \mathrm{hPa}$.

From above the planetary boundary layer to the upper stratosphere, the mechanical acceleration term $\bar{M}$ in Eq. (1) is principally due to planetary-wave forcing. In the TEM framework, this forcing is well approximated by the divergence of the E-P flux,

$\bar{M} \approx \frac{1}{a \cos \phi}\left[\frac{1}{a \cos \phi} \frac{\partial\left(F^{\phi} \cos \phi\right)}{\partial \phi}+\frac{\partial F^{p}}{\partial p}\right]$,

where $\mathrm{p}$ denotes pressure, and $F^{\phi}$ and $F^{p}$ denote the two components of the E-P flux vector $\boldsymbol{F}$ (e.g., Peixoto and Oort, 1992). We performed our analysis at $60^{\circ} \mathrm{N}$, since planetarywave forcing is known to reach a maximum there (Christiansen, 2001), which increases the validity of our assumption that $\bar{M}$ is well-represented by Eq. (4).

For each of the six November to April seasons, we have calculated the Fourier transforms of $\partial \bar{u} / \partial t, f \overline{v^{*}}$, and $\bar{M}$ separately. The six independent power spectra estimates were subsequently averaged at each frequency. By taking the square root of the ratio between the power estimates for $\partial \bar{u} / \partial t$ and $\bar{M}$, an estimate was obtained for the frequencydependent response of $\partial \bar{u} / \partial t$ to the wave forcing $\bar{M}$. Subsequently, we performed a least-squares fit of the theoretical 


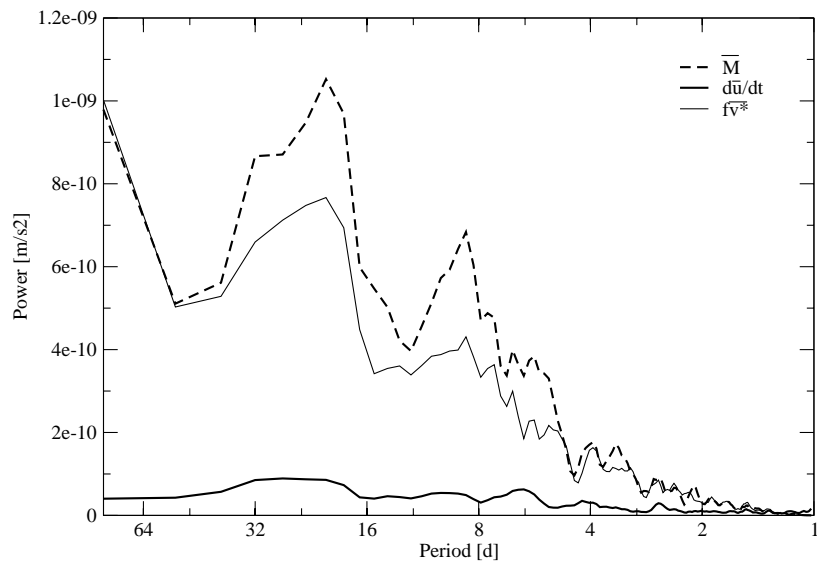

Fig. 1. Power spectrum estimates for the November-April season at $60^{\circ} \mathrm{N}$ for $\partial \bar{u} / \partial t$ (thick solid line), $f \overline{v^{*}}$ (thin solid line), and $\bar{M}$ (dashed line) at the $9.9 \mathrm{hPa}$ level. The spectral estimates have been smoothed using five-point averages.

response function $r$, defined in Eq. (3), to the ratios from the analyzed data. Here Eq. (3) is written as $r^{-2} \equiv a+b \omega^{-2}$, with $a \equiv\left(1+\mu^{-1}\right)^{2}$ and $b \equiv \alpha^{2} \mu^{-2}$. Estimating $a$ and $b$ then implies performing a linear least-squares regression analysis with $r^{-2}$ and $\omega^{-2}$ as known dependent $(y)$ and independent $(x)$ variables, respectively. With this spectral analysis, estimates for both $\alpha^{-1}$ and $\left(1+\mu^{-1}\right)^{-1}$ were obtained at all 35 pressure levels, where $\alpha^{-1} \equiv(\sqrt{a}-1) / \sqrt{b}$ and $\left(1+\mu^{-1}\right)^{-1} \equiv 1 / \sqrt{a}$. The goodness of fit is expressed by $R^{2}$, which is the square of the correlation coefficient of $r^{-2}$ and $\omega^{-2}$.

\section{Results}

Figure 1 shows the power spectrum estimates for the middle stratosphere at $9.9 \mathrm{hPa}$. This example is qualitatively representative for the stratosphere, with generally decreasing power for the forcing term $\bar{M}$ and the Coriolis term $f \overline{v^{*}}$ as the frequency increases. At timescales of 10-40 days, a broad maximum is found in the power spectra, which agrees with the sub-seasonal variability in the tropical upwelling that was found by Randel et al. (2002). At all frequencies, the estimate of $\left|\partial \bar{u}_{\omega} / \partial t\right|^{2}=\omega^{2}\left|\bar{u}_{\omega}\right|^{2}$ is clearly smaller than that of the forcing, roughly following the maxima and minima of the spectral power estimates for $f \overline{v^{*}}$ and $\bar{M}$, as expected from Eq. (3). The symbols in Fig. 2 show the (non-smoothed) frequency dependence of the estimated ratios $\left|\partial \bar{u}_{\omega} / \partial t\right| /\left|\bar{M}_{\omega}\right|$ at $0.1 \mathrm{hPa}, 9.9 \mathrm{hPa}$, and $1000 \mathrm{hPa}$. At all three levels, the ratio increases with increasing frequency, as expected from Eq. (3). The theoretical least-squares fits are based on the ratios at 45 angular frequencies, from $2 \times(2 \pi) \mathrm{yr}^{-1}$ to $90 \times(2 \pi) \mathrm{yr}^{-1}$, with steps of $2 \times(2 \pi) \mathrm{yr}^{-1}$. Higher frequencies, i.e., timescales shorter than about 4 days, are not included, since inconsistencies between subsequent

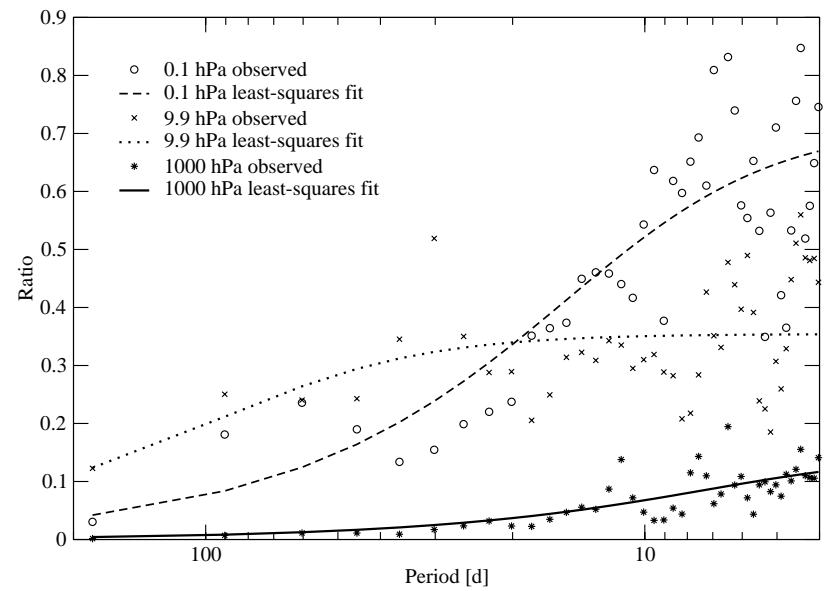

Fig. 2. Estimates (symbols) for the Nov-Apr season at $60^{\circ} \mathrm{N}$ for the ratio $\left|\partial \bar{u}_{\omega} / \partial t\right| /\left|\bar{M}_{\omega}\right|$ at $0.1 \mathrm{hPa}, 9.9 \mathrm{hPa}$, and $1000 \mathrm{hPa}$. Each line represents the least-squares fit of the theoretical response function to the observed ratios.
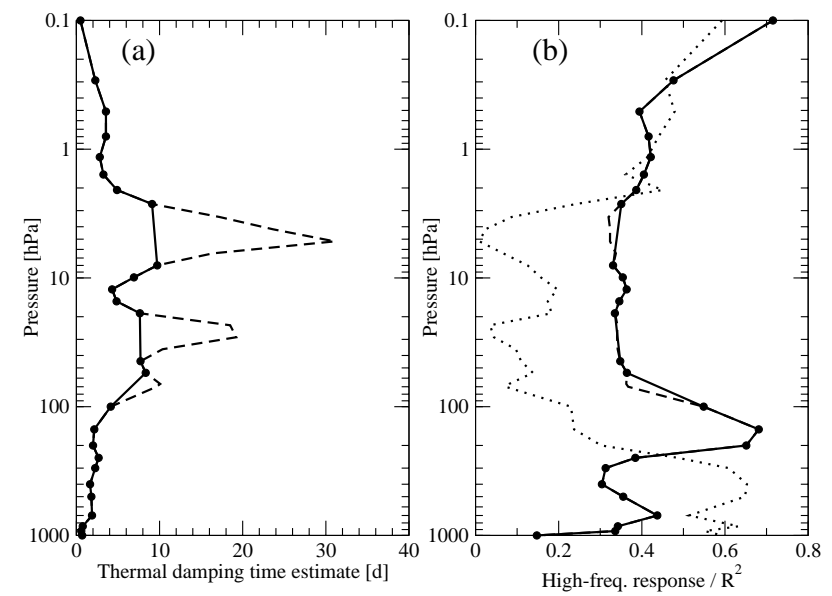

Fig. 3. Estimates for the November-April season at $60^{\circ} \mathrm{N}$ for (a) the thermal damping time $\alpha^{-1}$ and (b) the high-frequency limit of the response $\left(1+\mu^{-1}\right)^{-1}$, as a function of pressure. The dashed lines show the estimates at all levels, the solid lines only show the estimates at the levels where $\mathrm{R}^{2}$, the squared correlation coefficient of the least-squares regression, exceeds $10 \%$. The dotted line in (b) shows $R^{2}$ for the least-squares theoretical fit.

analyses lead to non-physical variability at high frequencies. The dashed lines in Figs. $3 \mathrm{a}$ and $\mathrm{b}$ show the computed vertical profiles of, respectively, the thermal damping time $\alpha^{-1}$ and the high-frequency limit $\left(1+\mu^{-1}\right)^{-1}$ of the response. The regression estimate of the thermal damping time is about 2 days in the troposphere (below $250 \mathrm{hPa}$ ), increasing to $7-$ 10 days in the stratosphere and decreasing to $2-4$ days in the lower mesosphere (above $1 \mathrm{hPa}$ ). The standard errors of these results are typically 4 days in the troposphere, 30 days in the stratosphere, and 10 days in the lower mesosphere. The standard errors become larger for longer thermal 
damping times, since the regression is then sensitive to the ratios at low frequencies, where the spectral resolution is relatively low. The sensitivity of $R^{2}$ (dotted line in Fig. 3b) to the thermal damping time estimate is clearly seen when comparing it with Fig. 3a. The solid lines in Figs. 3a and b show the profiles that only include points with $R^{2}>0.1$. The estimated stratospheric $\alpha^{-1}$ profile then becomes much smoother, whereas the $\left(1+\mu^{-1}\right)^{-1}$ estimate is less sensitive. We first compare our estimates of $\alpha^{-1}$ from the frequencydependent TEM model with those calculated by Newman and Rosenfield (1997, Fig. 3, hereafter NR97), who used a radiative transfer model to derive thermal damping times as a function of latitude, height, and season. The damping times found in our study are generally shorter than their estimates for November to April at $60^{\circ} \mathrm{N}$, but in the upper stratosphere, the agreement is quite good, especially considering the large stratospheric standard errors. In the lower stratosphere and troposphere however, our $\alpha^{-1}$ estimates are much smaller than those of NR97, who calculated wintertime damping times of 35-40 days at $100 \mathrm{hPa}$. Held and Suarez (1994) proposed a profile which for $60^{\circ} \mathrm{N}$ yields an $\alpha^{-1}$ of 26 days at $1000 \mathrm{hPa}$, linearly increasing to 40 days at $700 \mathrm{hPa}$ and above. With our tropospheric $\alpha^{-1}$ estimates of about 2 days and a standard error of about 5 days, the differences can be called significant. Concerning tropospheric thermal damping times, little consensus exists in the literature. This was also acknowledged by Burde (1999), who stated that $\alpha^{-1}$ is typically set to be the order of several days in the troposphere. Barry et al. (2000) deduced a radiative damping timescale of about 20 days in the troposphere by performing several GCM experiments in which radiative damping was turned off and back on. Prinn (1977) pointed out that, especially at low levels, thermal damping times will often be significantly overestimated if calculations do not include the effect of the conducting and radiating surface. Applying his analytical expression for $\alpha^{-1}$, tropospheric temperature perturbations with a vertical wavelength of about $3 \mathrm{~km}$ $\left(n^{-1}=0.5 \mathrm{~km}\right)$ at an average temperature $T_{0}=263 \mathrm{~K}$ are thermally damped (mainly by water vapor) with $\alpha^{-1}$ as short as 0.7 to 2.0 days. Therefore, the tropospheric thermal damping times found in this study lie just within the range of existing radiative timescale estimates. However, for the troposphere, it is difficult to justify a direct comparison between our estimates for the effective thermal damping time and the radiative damping times derived in other studies. The effective thermal damping time is reduced where thermal dissipation by turbulence and convection plays a role (e.g., Wu et al., 2000). This may explain why the effective damping times we find are on the (very) short end of the existing range of radiative damping time estimates, especially at low levels.

The high-frequency limit of the response (Fig. 3b) is generally quite constant with height and has a standard error of approximately 0.2 . In the stratosphere, at high frequencies, the ratio between $\left|\partial \bar{u}_{\omega} / \partial t\right|$ and $\left|\bar{M}_{\omega}\right|$ generally converges to $0.33-0.37$. Alternatively, $\mu$ converges to $0.5-0.6$, which is indeed $\mathrm{O}(1)$ as stated by Garcia (1987). The $\left(1+\mu^{-1}\right)^{-1}$ profile has a remarkable maximum near $150 \mathrm{hPa}$. This suggests that in the lowermost stratosphere, the variability of $\partial \bar{u} / \partial t$ is less dominated by angular momentum deposition by planetary waves, and more by conservation of absolute angular momentum. Power spectrum estimates at $150 \mathrm{hPa}$ (not shown) indicate that the variability of $\bar{M}$ is indeed small compared to that of both $\partial \bar{u} / \partial t$ and $f \overline{v^{*}}$. The rapid decrease of the $\left(1+\mu^{-1}\right)^{-1}$ estimate at the lowest levels is explained by the damping effect of surface drag on $\left|\partial \bar{u}_{\omega} / \partial t\right|$, which is not included in our calculation of $\bar{M}$.

\section{Discussion and conclusions}

In this paper, we have applied the frequency-dependent TEM model of Garcia (1987) to wind and temperature analyses from the ECMWF model. Six November-April seasons of 4-D-Var analysis data at 35 levels were used to derive power spectra for the planetary-wave forcing (E-P flux divergence), the zonal-mean zonal wind tendency, and the Coriolis acceleration associated with the residual meridional wind. We have used 4-D-Var data, since 3-D-Var analyses are known to yield a less realistic age of air in the stratosphere, which is mainly determined by the strength of the wave forcing (e.g., Scheele et al., 2005). We find that the theoretical response curve fits reasonably well onto the response derived from ECMWF analyses, with best agreement in the troposphere as well as in the upper stratosphere and lower mesosphere. Based on least-squares fits, estimates were obtained for the vertical profile of the effective thermal damping time and the high-frequency limit of the response. The analysis was performed at $60^{\circ} \mathrm{N}$, where the resolved planetary-wave forcing $\bar{M}$ reaches a maximum. The forcing, given by Eq. (4), does not incorporate the zonal momentum deposition due to breaking gravity waves, which is known to play an important role in the mesosphere. In addition, the forcing might be contaminated by physical inconsistencies in the data due to the assimilation process. To reduce the impact of these inconsistencies, timescales shorter than about 4 days were omitted from the analysis.

In this study, the scaling parameter $\mu$ is assumed to be a function of height only. However, Holton et al. (1995) argued from a scaling analysis of the vertical mass flux equation, that $\mu$ should have a particular frequency dependence, to allow for a non-zero vertical mass flux outside the forcing area. Based on their scaling arguments outside the forcing region, we obtain $\mu \sim \sqrt{1+\alpha^{2} / \omega^{2}} \max \left[1, L_{z} / H\right]$, where $H \sim 7 \mathrm{~km}$ is the mean scale height. This yields an aspect ratio of $L_{y} / L_{z} \leq N / f$ for all frequencies. However, with our analysis well inside the forcing region, we find an aspect ratio that is in general about $35 \%$ larger than $N / f$. Only in the lower stratosphere, where the forcing $\left|\bar{M}_{\omega}\right|$ is relatively weak, we find $L_{y} / L_{z} \leq N / f$. Our results therefore suggest that the aspect ratio for the response outside the forcing 
region as considered by Holton et al. (1995) is not applicable well inside the forcing region. For instance, due to vertical variations in the forcing field, the net meridional response within the forcing area may actually consist of multiple shallow meridional cells. This would yield a larger aspect ratio inside the forcing region, and a lower "effective" aspect ratio outside the forcing area, which still satisfies the condition $\left(L_{y} / L_{z}\right)_{\text {eff }} \leq N / f$. Furthermore, substituting $\mu$ in Eq. (2) leads to non-physical behavior at low frequencies, since the stationary limit $\omega \rightarrow 0$ yields a non-zero response between $\frac{1}{2} \sqrt{2}$ and 1 . Therefore, we have not applied these scaling arguments to $\mu$, although we have allowed it to vary with height instead of assuming a constant aspect ratio of $N / f$, as was assumed by Garcia (1987).

Although the agreement between our estimates of the thermal damping time and those of NR97 is quite good at higher levels, we found significantly shorter thermal damping times in the lower stratosphere and troposphere, where thermally dissipative processes other than radiative damping are important. This likely explains why our results lie on the very short end of the broad range of tropospheric radiative damping times found in the literature. Overall, the results suggest that Garcia's model for the frequency-dependent response of the zonal-mean flow to wave forcing is applicable to NH midlatitude wintertime conditions.

Acknowledgements. The authors are grateful to the editor and two anonymous reviewers for their helpful suggestions to improve the manuscript.

Edited by: P. Haynes

\section{References}

Barry, L., Craig, G. C., and Thuburn, J.: A GCM investigation into the nature of baroclinic adjustment, J. Atmos. Sci., 57, 11411155, 2000.

Burde, G. I.: Semigeostrophic Eady wave frontogenesis in the presence of Newtonian cooling, J. Atmos. Sci., 56, 3774-3788, 1999.

Christiansen, B.: Downward propagation of zonal mean zonal wind anomalies from the stratosphere to the troposphere, Model and reanalysis, J. Geophys. Res., 106(D21), 27 307-27 322, 2001.

Garcia, R. R.: On the mean meridional circulation in the middle atmosphere, J. Atmos. Sci., 44, 3599-3609, 1987.

Haynes, P. H.: Stratospheric dynamics, Ann. Rev. Fluid Mech., 37, 263-293, 2005.

Held, I. M. and Suarez, M. J.: A proposal for the intercomparison of the dynamical cores of atmospheric general circulation models, Bull. Am. Meteorol. Soc., 73, 1825-1830, 1994.

Holton, J. R., Haynes, P. H., McIntyre, M. E., Douglass, A. R., Rood, R. B., and Pfister, L.: Stratosphere-troposphere exchange, Rev. Geophys., 33, 403-439, 1995.

Limpasuvan, V., Thompson, D. W. J., and Hartmann, D. L.: The life cycle of the Northern Hemisphere sudden stratospheric warmings, J. Climate, 17, 2584-2596, 2004.

Newman, P. A. and Rosenfield, J. E.: Stratospheric thermal damping times, Geophys. Res. Lett., 24, 433-436, 1997.

Peixoto, J. P. and Oort, A. H.: Physics of climate, American Institute of Physics, New York, 520 pp., 1992.

Prinn, R. G.: On the radiative damping of atmospheric waves, J. Atmos. Sci., 34, 1386-1401, 1977.

Randel, W. J., Garcia, R. R., and Wu, F.: Time-dependent upwelling in the tropical lower stratosphere estimated from the zonal-mean momentum budget, J. Atmos. Sci., 59, 2141-2152, 2002.

Rosenlof, K. H.: Seasonal cycle of the residual mean meridional circulation in the stratosphere, J. Geophys. Res., 100(D3), 5173 5191, 1995.

Scheele, M. P., Siegmund, P. C., and Van Velthoven, P. F. J.: Stratospheric age of air computed with trajectories based on various 3 D-Var and 4-D-Var data sets, Atmos. Chem. Phys., 5, 1-7, 2005, http://www.atmos-chem-phys.net/5/1/2005/.

Shepherd, T. G. and Shaw, T. A.: The angular momentum constraint on climate sensitivity and downward influence in the middle atmosphere, J. Atmos. Sci., 61, 2899-2908, 2004.

Wu, Z., Battisti, D. S., and Sarachik, E. S.: Rayleigh friction, Newtonian cooling, and the linear response to steady tropical heating, J. Atmos. Sci., 57, 1937-1957, 2000. 